\title{
FUNCTIONS AND REGULARITY OF TOPOLOGICAL SPACES
}

\author{
ZBIGNIEW DUSZYŃSKI
}

Abstract. Preservation of regularity, almost regularity, and s-regularity of topological spaces, is considered.

\section{Introduction}

It is known that regularity is preserved under continuous closed surjections with compact point inverses (i.e., under perfect functions). In 1978, Noiri [19] proved that if $f:(X, \tau) \rightarrow$ $(Y, \sigma)$ is a continuous semi-closed surjection with compact point inverses, and $(X, \tau)$ is regular, then $(Y, \sigma)$ is $s$-regular. Murdeshwar [15] showed that regularity is preserved under surjections that are open, continuous, and closed. In 1986, Greenwood and Reilly gave a certain generalization of the Murdeshwar's result. In this paper we, in turn, generalize the Greenwood and Reilly's result [7, Proposition 8]. Moreover, we propose a number of further theorems related to preservation of regularity, almost regularity, and $s$-regularity of spaces, under surjections.

\section{Preliminaries}

Throughout, $(X, \tau)$ (and $(Y, \sigma))$ means a topological space (briefly: a space). Let $S \subset X$ be a subset of a space $(X, \tau)$. The closure of $S$ and the interior of $S$ will be denoted by $\operatorname{cl}_{\tau}(S)$ and $\operatorname{int}_{\tau}(S)$ (or simply $\operatorname{cl}(S)$ and $\operatorname{int}(S)$ ), respectively. The set $S$ is said to be regular open (resp. $\alpha$-open [16]; semi-open [10]; preopen [13]) in $(X, \tau)$, if $S=\operatorname{int}(\operatorname{cl}(S))(\operatorname{resp} . S \subset \operatorname{int}(\operatorname{cl}(\operatorname{int}(S)))$; $S \subset \operatorname{cl}(\operatorname{int}(S)) ; S \subset \operatorname{int}(\operatorname{cl}(S)))$. The $S$ is said to be $\alpha$-closed (resp. semi-closed [3]) in $(X, \tau)$, if $\operatorname{cl}(\operatorname{int}(\operatorname{cl}(S))) \subset S$ (resp. $\operatorname{int}(\operatorname{cl}(S)) \subset S)$. The collection of all regular open (resp. $\alpha$-open; semiopen; preopen) subsets of $(X, \tau)$ is denoted by $\operatorname{RO}(X, \tau)$ (resp. $\tau^{\alpha} ; \mathrm{SO}(X, \tau)$; $\left.\mathrm{PO}(X, \tau)\right)$. It is known that $\tau \subset \tau^{\alpha}$ (the equality does not hold, in general), and that $\tau^{\alpha}$ forms a topology on $X$. Obviously, $S$ is semi-closed in $(X, \tau)$ iff $X \backslash S$ is semi-open in $(X, \tau)$. Moreover, $S$ is semiclosed in $(X, \tau)$ iff $S=s \mathrm{cl}_{\tau}(S)$ [3, Theorem 1.4(2)], where $s \operatorname{cl}_{\tau}(S)$ (the semi-closed of $S$ in $(X, \tau)$ ) is defined analogously to the ordinary closrue. It is known that $\tau^{\alpha}=\operatorname{SO}(X, \tau) \cap \operatorname{PO}(X, \tau)[21$, Lemma 2.1].

Received March 23, 2007; revised September 23, 2007.

2000 Mathematics Subject Classification. 54C08.

Key words and phrases. Almost regular, $s$-regular spaces, almost open, $\alpha$-open, $\alpha$-closed, semi-closed functions, almost continuity, $\mathscr{R}$-mapness, $\alpha$-irresolutness. 
A function $f:(X, \tau) \rightarrow(Y, \sigma)$ is called almost open in the sense of Wilansky (briefly a.o.W.) [27, for injections], [20, Definition 1.3], if $f^{-1}\left(\mathrm{cl}_{\sigma}(V)\right) \subset \mathrm{cl}_{\tau}\left(f^{-1}(V)\right)$ for every $V \in \sigma$. In 1984, Rose [24, Theorem 11] has proved that $f$ if a.o.W. iff $f(U) \in \operatorname{PO}(Y, \sigma)$ for every $U \in \tau$ (i.e., iff $f$ is preopen [13]. A function $f:(X, \tau) \rightarrow(Y, \sigma)$ is called almost open in the sense of the Singals (briefly a.o.S.) [24], if $f(U) \in \sigma$ for every $U \in \operatorname{RO}(X, \tau)$. A function $f$ is said to be $\alpha$-open [14] (resp. pre- $\alpha-\boldsymbol{o p e n}$; s-open [1]), if $f(U) \in \sigma^{\alpha}$ (resp. $f(U) \in \sigma^{\alpha} ; f(U) \in \sigma$ ) for any set $U \in \tau$ (resp. $U \in \tau^{\alpha} ; U \in \operatorname{SO}(X, \tau)$ ). A function $f$ is called $\alpha$-closed [14] (resp. pre- $\alpha$-closed [5]; semi-closed [17]; pre-semi-closed [26]), if $f(U)$ is an $\alpha$-closed (resp. an $\alpha$-closed; a semiclosed; a semi-closed) subset of $(Y, \sigma)$ for each closed (resp. $\alpha$-closed; closed; semi-closed) subset $U$ of $(X, \tau)$.

A function $f:(X, \tau) \rightarrow(Y, \sigma)$ is almost continuous in the sense of the Singals (briefly a.c.S.) [24] (resp. an $\mathscr{R}$-map [2]; $\alpha$-irresolute [12]), if the preimage $f^{-1}(V) \in \tau$ [24, Theorem 2.2(b)] (resp. $f^{-1}(V) \in \operatorname{RO}(X, \tau) ; f^{-1}(V) \in \tau^{\alpha}$ ) for every set $V \in \operatorname{RO}(Y, \sigma)$ (resp. $V \in$ $\left.\mathrm{RO}(Y, \sigma) ; V \in \sigma^{\alpha}\right)$. Every $\mathscr{R}$-map is a.c.S., but the converse is false in general [22, Example 4.14]. It is known that $\alpha$-irresoluteness and continuity are independent notions [12, Examples $1 \& 2$ ].

A space $(X, \tau)$ is called extremally disconnected (briefly e.d.) if $\operatorname{cl}(S) \in \tau$ for every $S \in \tau$. A space $(X, \tau)$ is e.d. iff $\tau^{\alpha}=\mathrm{SO}(X, \tau)$ [8, Theorem 2.9].

A space $(X, \tau)$ is called almost regular [25, Theorem 2.2(b)] (resp. s-regular [11, Theorem 2(b)] if for each point $x \in X$ and each set $V \in \operatorname{RO}(X, \tau)$ ) (resp. $V \in \tau$ ) containing $x$, there exists a set $U \in \operatorname{RO}(X, \tau)$ (resp. $U \in \mathrm{SO}(X, \tau)$ ) such that $x \in U \subset \operatorname{cl}(U) \subset V$ (resp. $x \in U \subset \operatorname{scl}(U) \subset V$ ). Every regular space is almost regular (resp. $s$-regular) and the converse is not always true [18, Remark 1] (resp. [11, Example 1]). Conditions of almost regularity and $s$-regularity are independent of each other [11, Examples 1 and 2]. Recall that $(X, \tau)$ is called semi-regular if for each $x \in X$ and each $V \in \tau$ with $x \in V$, there exists a set $U \in \operatorname{RO}(X, \tau)$ such that $x \in U \subset V$.

\section{Almost regular spaces}

Theorem 1. [7, Proposition 8]. If $f:(X, \tau) \rightarrow(Y, \sigma)$ is an open, continuous, and $\alpha$-closed surjection, and $(X, \tau)$ is regular, then $(Y, \sigma)$ is regular.

It is clear that for any bijection $f:(X, \tau) \rightarrow(Y, \sigma)$, the properties of being $\alpha$-closed and being $\alpha$-open are equivalent. Thus, Theorem 1 leads to the well known result: every homeomorphism preserves the regularity property.

In order to generalize Theorem 1 we need the following lemma, which is, in turn, a generalization of [7, Lemma 2].

Lemma 1. Let $(X, \tau)$ be a space, $U \in \mathrm{PO}(X, \tau)$ and $U \subset V$. Then $\operatorname{cl}(U) \subset \operatorname{cl}(\operatorname{int}(\operatorname{cl}(V)))$.

Proof. We have $U \subset \operatorname{int}(\operatorname{cl}(U))$, whence as $\operatorname{int}(\operatorname{cl}(U)) \subset \operatorname{int}(\operatorname{cl}(V))$ we get $\operatorname{cl}(U) \subset \operatorname{cl}(\operatorname{int}(\operatorname{cl}(V)))$.

Theorem 2. Let a functon $f:(X, \tau) \rightarrow(Y, \sigma)$ be an a.o.W., continuous, and $\alpha$-closed surjection. If $(X, \tau)$ is regular, then $(Y, \sigma)$ is regular. 
Proof. Let $y \in Y$ and $U \in \sigma, U \ni y$, be arbitrary, and let $x \in X$ be such that $f(x)=y$. By regularity of $(X, \tau)$ there exists a set $G \in \tau$ such that $x \in G \subset \operatorname{cl}_{\tau}(G) \subset f^{-1}(U)$. Then $y \in$ $f(G) \subset f\left(\operatorname{cl}_{\tau}(G)\right) \subset U$. But, $f$ is a.o.W. and so, by Lemma $1, f(G) \subset \operatorname{int}_{\sigma}\left(\operatorname{cl}_{\sigma}(f(G))\right) \subset \operatorname{cl}_{\sigma}(f(G)) \subset$ $\operatorname{cl}_{\sigma}\left(\operatorname{int}_{\sigma}\left(\operatorname{cl}_{\sigma}\left(f\left(\operatorname{cl}_{\sigma}(G)\right)\right)\right)\right)$. Put $V=\operatorname{int}_{\sigma}\left(\operatorname{cl}_{\sigma}(f(G))\right) \in \operatorname{RO}(Y, \sigma)$. We obtain $y \in V \subset \operatorname{cl}_{\sigma}(V) \subset U$. This shows that $(Y, \sigma)$ is regular.

Corollary 1. Let a bijection $f:(X, \tau) \rightarrow(Y, \sigma)$ be $\alpha$-open and continuous. If $(X, \tau)$ is regular, then so is $(Y, \sigma)$.

Proof. Follows from Theorem 2. Just use the fact that each $\alpha$-open mapping is a.o.W.

An analysis of the proof of Theorem 2 leads to Theorems 3 and 4 below. The details are left to the reader.

Theorem 3. Let $f:(X, \tau) \rightarrow(Y, \sigma)$ be an a.o.W., a.c.S., and $\alpha$-closed surjection. If $(X, \tau)$ is regular, then $(Y, \sigma)$ is almost regular.

Corollary 2. Let a bijection $f:(X, \tau) \rightarrow(Y, \sigma)$ be $\alpha$-open and a.c.S. If $(X, \tau)$ is regular, then $(Y, \sigma)$ is almost regular.

Theorem 4. Let a surjection $f:(X, \tau) \rightarrow(Y, \sigma)$ be an a.o.S. and $\alpha$-closed $\mathscr{R}$-map. If $(X, \tau)$ is almost regular, then $(Y, \sigma)$ is almost regular.

Corollary 3. Assume a bijection $f:(X, \tau) \rightarrow(Y, \sigma)$ is an open $\mathscr{R}$-map. If $(X, \tau)$ is almost regular, then $(Y, \sigma)$ is almost regular.

Noiri has stated [20, Examples 1.6 \& 1.7] that a.o.W. and a.o.S. are independent notions. The following examples show that these notions remain independent if considered within the class of bijective mappings.

Example 1. (a). Let $X=\{a, b, c\}, \tau=\{\varnothing, X,\{a\},\{b\},\{a, b\}\}, \sigma=\{\varnothing, X\}$. The identity id: $(X, \tau) \rightarrow(X, \sigma)$ is a.o.W. and not a.o.S.

(b). Let $X=\{a, b\}, \tau=\{\varnothing, X,\{a\}\}, \sigma=\{\varnothing, X,\{b\}\}$. Then: id: $(X, \tau) \rightarrow(X, \sigma)$ and id ${ }^{-1}$ are a.o.S., but not a.o.W.

Now, we provide an example of bijection $f:(X, \tau) \rightarrow(X, \sigma)$ which is continuous (and so a.c.S.), but is not an $\mathscr{R}$-map (compare this example to [22, Example 4.14] for the non-bijective case).

Example 2. Let $X=\{a, b, c\}, \tau=\{\varnothing, X,\{b\},\{c\},\{a, b\},\{b, c\}\}, \sigma=\{\varnothing, X,\{a\},\{b\},\{a, b\}\}$. Let $f:(X, \tau) \rightarrow(X, \sigma)$ be defined as follows: $f(a)=c, f(b)=a, f(c)=b$. Then $f$ is not an $\mathscr{R}$-map singce $f^{-1}(\{a\}) \notin \operatorname{RO}(X, \tau)$.

$\mathscr{R}$-mapness and continuity are independent notions, even in the class of bijections. Because of Example 2, it is enough to indicate an $\mathscr{R}$-map which is not continuous.

Example 3. Let $X=\{a, b\}, \tau=\{\varnothing, X\}, \sigma=\{\varnothing, X,\{a\}\}$. The identity id: $(X, \tau) \rightarrow(X, \sigma)$ is an $\mathscr{R}$-map which is not continuous. 
Remark 1. An a.c.S. functon (even a bijection) need not be continuous (compare the following example to [24, Example 2.2]) nor an $\mathscr{R}$-map. Let $X=\{a, b, c, d\}, \tau=\{\varnothing, X,\{a\},\{b\},\{a, b\},\{b, c\},\{a, b, c\}\}$, $\sigma=\{\varnothing, X,\{b\},\{c\},\{b, c\},\{b, c, d\}\}$, and let $f(a)=c, f(b)=b, f(c)=a, f(d)=d$.

Theorem 5. Let $(X, \tau)$ be a space. If $\left(X, \tau^{\alpha}\right)$ is regular, then $(X, \tau)$ is regular.

Proof. Assume $\left(X, \tau^{\alpha}\right)$ is regular. Suppose $x \in X$ and $U \in \tau$ such that $x \in U$, are arbitrary. By the hypothesis, there is a set $V \in \tau^{\alpha}$ such that $x \in V \subset \operatorname{cl}_{\tau^{\alpha}}(V) \subset U . \operatorname{But} \operatorname{cl}_{\tau^{\alpha}}(V)=\operatorname{cl}_{\tau}(V)[6$, Lemma 1(i)] and as $V \in \mathrm{PO}(X, \tau)$ we get $x \in V \subset \operatorname{int}_{\tau}\left(\operatorname{cl}_{\tau}(V)\right) \subset \operatorname{cl}_{\tau}\left(\operatorname{int}_{\tau}\left(\operatorname{cl}_{\tau}(V)\right)\right) \subset \mathrm{cl}_{\tau}(V)$. This shows $(X, \tau)$ is regular.

The converse to Theorem 5 may not be true as the following example shows.

Example 4. Consider the real line $\mathbb{R}$ endowed with the Euclidean topology $\tau_{e}$. The space $\left(\mathbb{R}, \tau_{e}^{\alpha}\right)$ is not regular: take into account $x=0$ and its $\alpha$-neighbourhood $V=[-1,1] \backslash \bigcup_{i=1}^{\infty}\left\{-\frac{1}{i}, \frac{1}{i}\right\}$.

Lemma 2. $[9$, Corollary 2.4(a) $] . \mathrm{RO}(X, \tau)=\mathrm{RO}\left(X, \tau^{\alpha}\right)$ for each space $(X, \tau)$.

Theorem 6. A space $(X, \tau)$ is almost regular if and only if $\left(X, \tau^{\alpha}\right)$ is alnost regular.

Proof. Clear by Lemma 2 and the fact that $\operatorname{cl}_{\tau}(S)=\operatorname{cl}_{\tau^{\alpha}}(S)$ for each $S \in \mathrm{SO}(X, \tau)[6$, Lemma 1(i)].

Definition 1. A function $f:(X, \tau) \rightarrow(Y, \sigma)$ is $\alpha$-a.o.W. if $f(U) \in \operatorname{PO}(Y, \sigma)$ for every $U \in \tau^{\alpha}$.

Obviously, each $\alpha$-a.o.W. function is a.o.W. The converse implication does not hold.

Example 5. Let $X=\{a, b, c\}, \tau=\{\varnothing, X,\{a\}\}, \sigma=\{\varnothing, X,\{a\},\{b\},\{a, b\}\}$. The identity id: $(X, \tau) \rightarrow$ $(X, \sigma)$ is open (and hence a.o.W.), but it is not $\alpha$-a.o.W. because $\operatorname{id}(\{a, c\}) \notin \operatorname{PO}(X, \sigma)$.

Lemma 3. Let $(X, \tau)$ be a space. Then,

(1) $\left[9\right.$, Corollary 2.5(a)]. $\mathrm{PO}(X, \tau)=\mathrm{PO}\left(X, \tau^{\alpha}\right)$;

(2) [16, Proposition 10]. $\tau^{\alpha}=\left(\tau^{\alpha}\right)^{\alpha}$.

Theorem 7. Let a surjection $f:(X, \tau) \rightarrow(Y, \sigma)$ be $\alpha$-a.o.W. $\alpha$-irresolute, pre- $\alpha$-closed. If $\left(X, \tau^{\alpha}\right)$ is regular then $\left(Y, \sigma^{\alpha}\right)$ is regular.

Proof. By Lemma 3, the surjection $f_{\star}:\left(X, \tau^{\alpha}\right) \rightarrow\left(Y, \sigma^{\alpha}\right)$, where $f_{\star}(x)=f(x)$ for each $x \in X$ (see [9, page 86, line 11]), is also a.o.W., continuous, and $\alpha$-closed. Thus by Theorem 2 we get $\left(Y, \sigma^{\alpha}\right)$ is regular.

Since every bijection is pre- $\alpha$-closed if and only if it is pre- $\alpha$-open, one obtains the well known result for homeomorphisms: $\left(X, \tau^{\alpha}\right) \rightarrow\left(Y, \sigma^{\alpha}\right)$ (the one stated right under Theorem 1$)$.

Recall that a function $f:(X, \tau) \rightarrow(Y, \sigma)$ is a semi-homeomorphism [4] if it is bijective, pre-semi-open $(f(U) \in \mathrm{SO}(Y, \sigma)$ for every $U \in \mathrm{SO}(X, \tau))$, and irresolute $\left(f^{-1}(V) \in \mathrm{SO}(X, \tau)\right.$ for every $V \in \mathrm{SO}(Y, \sigma))$. The following result is known: a function $f:(X, \tau) \rightarrow(Y, \sigma)$ is a semihomeomorphism if and only if $f_{\star}:\left(X, \tau^{\alpha}\right) \rightarrow\left(Y, \sigma^{\alpha}\right)$ is a semi-homeomorphism $[6$, Theorem $1]$. 
Corollary 4. Let a function $f:(X, \tau) \rightarrow(Y, \sigma)$ be a semi-homeomorphism. If $\left(X, \tau^{\alpha}\right)$ is regular, then $\left(Y, \sigma^{\alpha}\right)$ is also regular.

This corollary is interesting in regard for Theorem 5 and Example 4 above, and the fact that the image of a regular space under a semi-homeomorphism is not necessarily regular [4, Example 1.5]. Moreover, by Corollary 4 and Theorem 5, [4, Example 1.5] is another illustration that regularity of $(X, \tau)$ need not imply regularity of $\left(X, \tau^{\alpha}\right)$.

The next theorem is a simple consequence of Theorems 3,5 , and 6 .

Theorem 8. Let $f:(X, \tau) \rightarrow(Y, \sigma)$ be an a.o.W., a.c.S., and $\alpha$-closed surjection. If $\left(X, \tau^{\alpha}\right)$ is regular, then $\left(Y, \sigma^{\alpha}\right)$ is almost regular.

Corollary 5. Let a bijection $f:(X, \tau) \rightarrow(Y, \sigma)$ be $\alpha$-open and a.c.S. If $\left(X, \tau^{\alpha}\right)$ is regular, then $\left(Y, \sigma^{\alpha}\right)$ is almost regular.

Proof. Follows from Theorem 8.

In the class of bijective mappings, $\alpha$-irresoluteness and continuity are independent of each other [12, Examples $1 \& 2$ ].

Example 6. (a). Let $X=\{a, b, c, d\}, \tau=\{\varnothing, X,\{a\},\{b, c\},\{a, b, c\}\}, \sigma=\{\varnothing, X,\{a\}\}$. The identity id: $(X, \tau) \rightarrow(X, \sigma)$ is continuous (hence a.c.S.) and not $\alpha$-irresolute, since id ${ }^{-1}(\{a, b\}) \notin \tau^{\alpha}$.

(b). Let $X=\{a, b, c, d\}, \tau=\{\varnothing, X,\{a\},\{b\},\{a, b\},\{b, c\},\{a, b, c\}\}, \sigma=\tau^{\alpha}=\tau \cup\{\{a, b, c\}\}$. Then id: $(X, \tau) \rightarrow(X, \sigma)$ is discontinuous but $\alpha$-irresolute.

Problem 1. Find an $\alpha$-irresolute bijection (or just a surjection) which is not a.c.S.

Theorem 9. Let $f:(X, \tau) \rightarrow(Y, \sigma)$ be a surjective $\alpha$-closed and a.o.S. $\mathscr{R}$-map. If $\left(X, \tau^{\alpha}\right)$ is regular, then $\left(Y, \sigma^{\alpha}\right)$ is almost regular.

Proof. We apply Theorems 5, 4, and 6, respectively.

Corollary 6. Let a bijection $f:(X, \tau) \rightarrow(Y, \sigma)$ be an open $\mathscr{R}$-map. If $\left(X, \tau^{\alpha}\right)$ is regular then $\left(Y, \sigma^{\alpha}\right)$ is almost regular.

Comparing Theorems 7 and 9 it is worthy of notice that $\alpha$-a.o.W. and a.o.S. are independent notions, even within the class of bijections.

Example 7. (a). Let $X=\{a, b, c\}, \tau=\{\varnothing, X,\{a\},\{b\},\{a, b\}\}, \sigma=\{\varnothing, X\}$. Then, id: $(X, \tau) \rightarrow$ $(X, \sigma)$ is $\alpha$-a.o.W. and not a.o.S., because for $S=\{a\}$ (or $S=\{b\}), \operatorname{id}(S) \notin \sigma$.

(b). Let $X=\{a, b\}, \tau=\{\varnothing, X,\{a\}\}, \sigma=\{\varnothing, X,\{b\}\}$. The identity id: $(X, \tau) \rightarrow(X, \sigma)$ is a.o.S. and not $\alpha$-a.o.W.

In order to see that $\alpha$-irresoluteness and $\mathscr{R}$-mapness are independent of each other, it is enough to apply Examples 2 and 3.

\section{4. $s$-regular spaces}


It is well-known that regularity implies semi-regularity. Examples of spaces which are semi-regular and not regular are also known [25, Remark 3.2] and [11, Example 1].

Theorem 10. If a space $(X, \tau)$ is semi-regular, then it is s-regular.

Proof. We shall use [9, Proposition 2.7(a)]: $s \operatorname{cl}(S)=\operatorname{int}(\operatorname{cl}(S))$ for any $S \in \operatorname{PO}(X, \tau)$. Let $x \in X$ and $U \in \tau$ be such that $x \in U$. By hypothesis there exists a set $V \in \operatorname{RO}(X, \tau)$ with $x \in V \subset U$. So, $x \in \operatorname{int}(\operatorname{cl}(V))=\operatorname{scl}(V) \subset U$.

Problem 2. One does not know an example of $s$-regular space that fails to be semi-regular.

Theomre 11. Let an $(X, \tau)$ be e.d. The following conditions are equivalent:

(a) $(X, \tau)$ is regular,

(b) $(X, \tau)$ is semi-regular,

(c) $(X, \tau)$ is s-regular.

Proof. Since the implication (b) $\Rightarrow$ (a) is clear by the definition of e.d. space, only (c) $\Rightarrow(b)$ requires a proof. Let $x \in X$ and $U \in \tau$ be such that $x \in U$. By hypothesis, there exists a set $G \in$ $\operatorname{SO}(X, \tau)$ with $x \in G \subset \operatorname{scl}(G) \subset U$. Since $\operatorname{int}(\operatorname{cl}(S)) \subset \operatorname{scl}(S)$ for any $S \subset X$ [21, Lemma 4.14] and $\mathrm{SO}(X, \tau) \subset \mathrm{PO}(X, \tau)$ [9, Proposition 4.1], one obtains $x \in V \subset \operatorname{int}(\operatorname{cl}(V)) \subset U$ for $V=\operatorname{int}(\operatorname{cl}(G))$. Therefore $(X, \tau)$ is semi-regular.

Notice that $s$-regularity and e.d. are independent one of the other [11, Examples 1 and 2]. Recall also, that there is an almost regular and e.d. space which is not $s$-regular [11, Example 2], and a semi-regular not e.d. space which is almost regular [11, Example 1].

Theorem 12. Let a surjection $f:(X, \tau) \rightarrow(Y, \sigma)$ be $\alpha$-a.o.W., continuous, and semi-closed. If $(X, \tau)$ is s-regular and e.d., then $(Y, \sigma)$ is semi-regular.

Proof. Let $y \in Y$ and $U \in \sigma$ with $y \in U$. Let for an $x \in X, y=f(x)$. By $s$-regularity of $(X, \tau)$ we have $x \in V \subset s \mathrm{sl}_{\tau}(V) \subset f^{-1}(U)$ for a certain $V \in \mathrm{SO}(X, \tau)$. Hence $y \in f(V) \subset f\left(s \mathrm{cl}_{\tau}(V)\right) \subset U$. But $(X, \tau)$ is e.d., so by [8, Theorem 2.9], $\operatorname{scl}_{\tau}(V)=\operatorname{cl}_{\tau^{\alpha}}(V)$. On the other hand $\operatorname{cl}_{\tau^{\alpha}}(V)=\operatorname{cl}_{\tau}(V)[6$, Lemma 1(i)]. As $f$ is semi-closed, $\operatorname{int}_{\sigma}\left(\operatorname{cl}_{\sigma}(f(V))\right) \subset s \mathrm{sl}_{\sigma}\left(f\left(\operatorname{int}_{\tau}\left(\mathrm{cl}_{\tau}\left(\operatorname{int}_{\tau}(V)\right)\right)\right)\right) \subset f\left(\mathrm{cl}_{\tau}(V)\right)$; [21, Lemma 4.14] and [8, Theorem 2.9]. Put $G=\operatorname{int}_{\sigma}\left(\operatorname{cl}_{\sigma}(f(V))\right)$. Thus $y \in G \subset \operatorname{int}_{\sigma}\left(\operatorname{cl}_{\sigma}(G)\right) \subset U$, because $f$ is $\alpha$-a.o.W. This completes the proof.

Definition 2. A mapping $f:(X, \tau) \rightarrow(Y, \sigma)$ is $s$-a.o.W. if $f(U) \in \operatorname{PO}(Y, \sigma)$ for every $U \in$ $\mathrm{SO}(X, \tau)$.

Obviously, each $s$-a.o.W. mapping is $\alpha$-a.o.W. The converse is false, even for bijections.

Example 8. Let $X=\{a, b, c, d\}, \sigma=\{\varnothing, X,\{a\},\{b, c\},\{a, b, c\}\}, \tau=\sigma \cup\{\{b\},\{a, b\}\}$. Then, id: $(X, \tau) \rightarrow(X, \sigma)$ is $\alpha$-a.o.W. and not $s$-a.o.W., because $\operatorname{id}(\{a, d\}) \notin \mathrm{PO}(x, \sigma)$.

We notice that $\alpha$-a.o.W. with openness and $s$-a.o.W. with openness, are couples of independent notions; see the examples below. 
Example 9. (a). Let $X=\{a, b, c\}, \tau=\{\varnothing, X,\{a\},\{b\},\{a, b\}\}, \sigma=\{\varnothing, X\}$. The identity from $(X, \tau)$ to $(X, \sigma)$ is $s$-a.o.W., but it is not open.

(b). Let $X=\{a, b, c\}, \tau=\{\varnothing, X,\{a\}\}, \sigma=\{\varnothing, X,\{a\},\{b\},\{a, b\}\}$. The identity from $(X, \tau)$ to $(X, \sigma)$ is open and not $\alpha$-a.o.W., since $\operatorname{id}(\{a, c\}) \notin \mathrm{PO}(X, \sigma)$.

Theorem 13. Let a surjection $f:(X, \tau) \rightarrow(Y, \sigma)$ be s-a.o.W., continuous, and pre-semiclosed. If $(X, \tau)$ is s-regular, then $(Y, \sigma)$ is semi-regular.

Proof. Let $y \in Y$ and $U \in \sigma$ with $y \in U$, and let $x \in X$ be such that $f(x)=y$. As $(X, \tau)$ is $s$-regular, there exists a set $V \in \mathrm{SO}(X, \tau)$ such that $x \in V \subset s \mathrm{l}_{\tau}(V) \subset f^{-1}(U)$. So, $y \in f(V) \subset$ $f\left(s \mathrm{l}_{\tau}(V)\right) \subset U$. From pre-semi-closedness of $f$ and from [21, Lemma 4.14] it follows that

$$
\operatorname{int}_{\sigma}\left(\operatorname{cl}_{\sigma}\left(f\left(\operatorname{int}_{\tau}\left(\mathrm{cl}_{\tau}(V)\right)\right)\right)\right) \subset \operatorname{int}_{\sigma}\left(\operatorname{cl}_{\sigma}\left(f\left(s \mathrm{cl}_{\tau}(V)\right)\right)\right) \subset f\left(s \mathrm{sl}_{\tau}(V)\right) .
$$

Continuity of $f$ implies $f\left(\operatorname{cl}_{\tau}\left(\operatorname{int}_{\tau}\left(\operatorname{cl}_{\tau}(V)\right)\right)\right) \subset \operatorname{cl}_{\sigma}\left(f\left(\operatorname{int}_{\tau}\left(\operatorname{cl}_{\tau}(V)\right)\right)\right)$. But, $f$ is $s$-a.o.W. and thus

$$
f(V) \subset \operatorname{int}_{\sigma}\left(\operatorname{cl}_{\sigma}(f(V))\right) \subset \operatorname{int}_{\sigma}\left(\operatorname{cl}_{\sigma}\left(f\left(\operatorname{cl}_{\tau}\left(\operatorname{int}_{\tau}\left(\mathrm{cl}_{\tau}(V)\right)\right)\right)\right) \subset \operatorname{int}_{\sigma}\left(\operatorname{cl}_{\sigma}\left(f\left(s \mathrm{cl}_{\tau}(V)\right)\right)\right) .\right.
$$

Put $G=\operatorname{int}_{\sigma}\left(\operatorname{cl}_{\sigma}\left(f\left(s \mathrm{cl}_{\tau}(V)\right)\right)\right)$. This shows $(Y, \sigma)$ is semi-regular.

Deinition 3. A function $f:(X, \tau) \rightarrow(Y, \sigma)$ is $s \alpha$-open if $f(U) \in \sigma^{\alpha}$ for every $U \in \operatorname{SO}(X, \tau)$.

Corollary 7. Let a bijection $f:(X, \tau) \rightarrow(Y, \sigma)$ be continuous and sa-open. If $(X, \tau)$ is $s$ regular, then $(Y, \sigma)$ is semi-regular.

Proof. By Theorem 13 and [21, Lemma 3.1].

Each $s$-open mapping is $s \alpha$-open, but the reverse implications does not hold in general, even for bijections.

Example 10. Let $X=\{a, b, c\}, \tau=\{\varnothing, X,\{a\},\{a, c\}\}, \sigma=\{\varnothing, X,\{a\}\}$. The identity from $(X, \tau)$ to $(X, \sigma)$ is $s \alpha$-open and not $s$-open (consider $\{a, b\} \in \operatorname{SO}(X, \tau)$, for instance).

\section{Acknowledgement}

The author is grateful to referee for his/her helpful suggestions and comments.

\section{References}

[1] D. E. Cameron and G. Woods, s-continuous and s-open mappings, preprint.

[2] D. A. Carnahan, Some properties related to compactness in topological spaces, Ph.D. thesis, University of Arkansas, 1973.

[3] C. G. Crossley and S. K. Hildebrand, Semi-closure, Texas J. Sci. 22(1971), 99-112.

[4] C. G. Crossley and S. K. Hildebrand, Semi-topological properties, Fundamenta Mathematicae 74(1972), 233-254. 
[5] Z. Duszyński, Some remarks on almost $\alpha$-continuous functions, Kyungpook Math. J. 44(2004), 249260.

[6] G. L. Garg and D. Sivaraj, Semitopological properties, Mat. Vesnik 36(1984), 137-142.

[7] S. Greenwood and I. L. Reilly, On feebly closed mappings, Indian J. Pure Appl. Math. 17(1986), 11011105.

[8] D. S. Janković, On locally irreducible spaces, Ann. Soc. Sci. Bruxelles 97(1983), 59-72.

[9] D. S. Janković, A note on mappings of extremally disconnected spaces, Acta Math. Hungar. 46(1985), 83-92.

[10] N. Levine, Semi-open sets and semi-continuity in topological spaces, Amer. Math. Monthly 70(1963), $36-41$.

[11] S. N. Maheshwari and R. Prasad, On s-regular spaces, Glasnik Mat. 10(1975), 347-350.

[12] S. N. Maheshwari and S. S. Thakur, On $\alpha$-irresolute mappings, Tamkang J. Math. 11(1980), 205-214.

[13] A. S. Mashhour, M. E. Abd El-Monsef and S. N. El-Deeb, On precontinuous and weak precontinuous mappings, Proc. Math. and Phys. Soc, Egypt 53(1982), 47-53.

[14] A. S. Mashhour, I. A. Hasanein and S. N. El-Deeb, $\alpha$-continuous and $\alpha$-open mappings, Acta Math. Hungar. 41(1983), 213-218.

[15] M. G. Murdeshwar, General Topology, Halstead Press, New York, 1983.

[16] O. Njåstad, On some classes of nearly open sets, Pacific J. Math. 15(1965), 961-970.

[17] T. Noiri, A generalization of closed mappings, Lincei-Rend. Sc. Fis. Mat. e Nat. 54(1971), 412-415.

[18] T. Noiri, A note an semi-regularizations, Glasnik Mat. 10(30)(1975), 141-143.

[19] T. Noiri, A note on s-regular spaces, Glasnik Mat. 13(33)(1978), 107-110.

[20] T. Noiri, Semi-continuity and weak-continuity, Czechoslovak Mathematical Journal 31(106)(1981), 314-321.

[21] T. Noiri, On $\alpha$-continuous functions, Čas. pěst. mat. 109(1984), 118-126.

[22] T. Noiri, Super-continuity and some strong forms of continuity, Indian J. Pure Appl. Math. 15(1984), 241-250.

[23] D. A. Rose, Weak continuity and almost continuity, Internat. J. Math. \& Math. Sci. 7(1984), 311-318.

[24] M. K. Singal and Asha Rani Singal, Almost-continuous mappings, Yokohama Math. J. 16(1968), 6373.

[25] M. K. Singal and Shashi Prabha Arya, On almost-regular spaces, Glasnik Mat. 4(24)(1969), 89-99.

[26] D. Sivaraj, Semihomeomorphisms, Acta Math. Hungar. 48(1986), 139-145.

[27] A. Wilansky, Topics in Functional Analysis, Lecture Notes in Mathematics 45, Springer-Verlag, 1967.

Institute of Mathematics, Casimirus the Great University, Pl. Weyssenhoffa 11, 85-072, Bydgoszcz, Poland. E-mail: imath@ukw.edu.pl 